\title{
Expert System Development through the Decision-Making Process and Optimization for Classifying Strategic Items
}

\author{
Dong Hoon Shin and Jae-Woong Tae
}

\begin{abstract}
International export control regimes such as Nuclear Suppliers Group strongly urge member states to control exports of strategic items used in the development, production, or use of WMD. South Korea is complying with these export control laws. There are two important rules within those laws: classification and export licensing. Classification means checking whether exported items are strategic items, based on export-control specifications. However, these specifications are sometimes ambiguous or qualitative. Thus, there are many problems in maintaining or securing consistency and objectivity in classifications. Here, we optimized classification through analyzing expert review processes, establishing review criteria, and developing a database to address these problems. Finally, we developed two expert systems for classification decisions. Because these systems are the first in the field of export control, we will use the systems for classification, and will improve the criteria and/or database to strengthen their performance.
\end{abstract}

Index Terms-Nuclear suppliers group, strategic items, export control, classification, expert system.

\section{INTRODUCTION}

International export control regimes such as the Nuclear Suppliers Group (NSG), the Missile Technology Control Regime (MTCR), the Wassenaar Arrangement (WA), and the Australia Group (AG) urge that member states control the export of strategic items that can be used for the development, production, or use of weapons of mass destruction (WMD), such as nuclear, biological, and chemical weapons. Taking NSG as a representative regime, related to nuclear weapons, the NSG published a guideline (IAEA Information Circular 254) and requests that member states comply with it [1]. So, the South Korean government established export control laws, such as the Foreign Trade Act [2] (Law) and the Minister's Regulation for the Export and Import of Strategic Goods (Minister's Regulation) [3] according to the NSG guideline, and has implemented the Law and Minister's Regulation.

There are two important rules - classification and export licensing-within the Law and Minister's Regulation. Classification means checking whether exported items are strategic items through comparing the exported items' specifications with the control specifications of the NSG guideline and the Minister's Regulation. Export licensing means granting the export of the strategic items through evaluating the influence on the proliferation of WMD and/or

Manuscript received November 20, 2014; revised February 25, 2015. This work was supported in part by Nuclear Safety and Security Commission (NSSC) and Korea Radiation Safety Foundation (KORSAFE).

The authors are with the Korea Institute of Nuclear nonproliferation and control (KINAC), 1534 Yuseong-daero, Yuseong-gu, Daejeon, 305-348, Republic of Korea (e-mail: nucleo@kinac.re.kr, tjwtjwtjw@kinac.re.kr). the reasonableness of the export.

However, there are some problems in the control language and specifications of the Minister's Regulation and NSG guideline that are used for classification. Typical problems are that the control language or specifications are ambiguous or qualitative. Because of these problems, the classification results often tend to differ by reviewers' experience, knowledge, or opinion for the same items. That is, different experts can reach different decisions for similar or the same items. This situation can harm the consistency and objectivity of classification results. So, we developed two expert systems, for physical items and technologies, to address these problems [4].

\section{Methodology AND Results}

The development procedure for the classification expert systems [4] is described in Fig. 1. It consisted of five steps, including classification process generalization, database development, and main review factors (MRFs), etc.

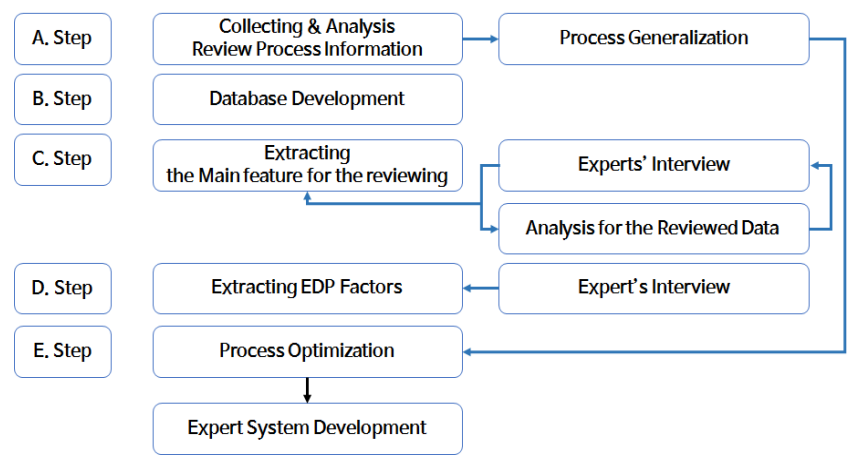

Fig. 1. Procedure for expert system development.

As shown in Fig. 1, the first step was generalizing the classification process through collecting and analyzing each expert's review process. The second step was building relevant databases that were used in each sub-step of the classification process [5]. The third step was extracting the important MRFs through expert interviews and re-analyzing previously reviewed documents [6]. The fourth step was extracting especially designed or prepared (EDP) factors (EFs) through expert interviews [6]. Then, the final step was optimizing the classification process and developing the expert systems.

\section{A. Generalization of the Classification Process}

As shown in Fig. 2, we first collected all of the step information related to review processes from the experts who were reviewing exported items, such as physical items and technologies. Second, we integrated all of the processes into a 
single process for physical items and technologies, and the sub-steps of the single process were merged or removed through eight experts' reviews for generalizing the classification process.

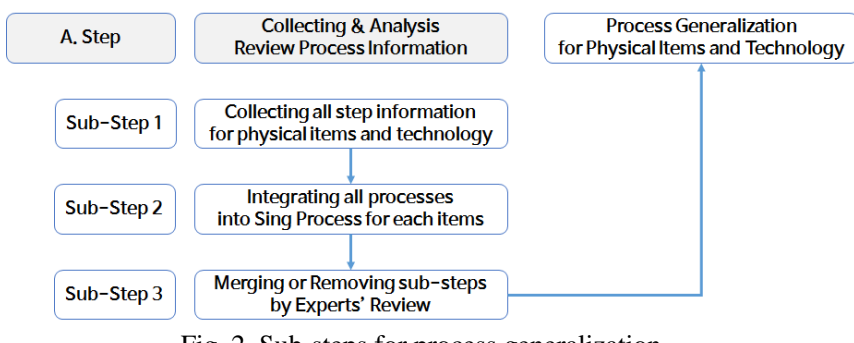

Fig. 2. Sub-steps for process generalization.

As a result of this step, we found that there were some differences between the classification of physical items and the classification of technologies. For example, the classification of physical items was composed of three sub-steps: item name matching, function matching, and specification matching when compared to the control specifications of the NSG guideline or the Minister's Regulation.

However, the classification of technologies involved four sub-steps: item name matching, open source checking, basic science checking, and technical level checking. Thus, three of the sub-steps for technology's classification were unique steps, different from those for physical items.

There was another important checking point or step in the classification process. That is, EDP checking, which means confirming that the reviewed items were especially or specifically prepared for the development, production, or use of nuclear systems. This EDP checking is a unique checking point: the main point of EDP is focused on the political aspects of the importing countries/companies, or the export's purpose.

\section{B. Database Development}

In the previous section, we generalized the classification processes for physical items and technologies, and found some unique characteristics for each process.

Database development is important, as is process optimization for the expert systems. Thus, we selected the necessary data for our systems, and reconstructed all of the data sources into appropriately structured data.

The necessary data sources for our expert systems were the Minister's Regulation, the NSG guideline, the NSG handbook [7], the system description books for nuclear power plants, and a terminology dictionary for the nuclear field. The Minster's Regulation and NSG guideline are the most important references, because these have information about the controlled items' names, control codes (e.g., 0A001, 0B001), and technical specifications. The NSG handbook and the system description books are references that contain vast amounts of information, such as system descriptions, system structures, technical specifications, usage, and the compositions of materials. Other references include dictionaries that contain terminology, abbreviations, and synonyms related to nuclear engineering.

In the next step, we developed a database from the selected data sources. We first compared the generalized processes with data sources, and selected the data sources needed in each sub-step of the generalized processes. Then, data sources were converted into the appropriate forms for the expert systems.

There were seven representative databases in our systems (Table I).

\begin{tabular}{cll}
\multicolumn{4}{c}{ TABLE I: DATABASE LIST FOR EXPERT SYSTEM } \\
\hline \hline No. & \multicolumn{1}{c}{ Database } & \multicolumn{1}{c}{ Explanatory } \\
\hline 1 & Primary database & $\begin{array}{l}\text { Core Database for Controlled Items } \\
\text { Public domain type } \\
\text {.com, .gov, .go.kr, .ac.kr } \\
\text { Basic science definition \& type } \\
\text { Technology definition \& sub } \\
\text { category }\end{array}$ \\
4 & URL database & Probability for Strategic Items \\
5 & $\begin{array}{l}\text { Strategic Probability } \\
\text { database }\end{array}$ & EDP Probability \\
6 & database & Probability related to EDP type \\
7 & Terminology database & Abbreviations, synonyms, etc. \\
\hline \hline
\end{tabular}

The primary database in Table I was the core database of the expert system. This contained all of the information noted in Table II. The second and third databases are a URL database and a basic science database; these were used to confirm whether the information of the reviewed items is public-domain information, and whether the information on the reviewed items can be categorized as basic science. The fourth database is a technology database; this is used to categorize the reviewed item into a specific technology group. The fifth and sixth databases are a strategic probability database and an EDP probability database; these are used for decision-making in the classification process. The detailed form and contents are described in Tables IV, V, and VI. The seventh database is a terminology database; this contains terminology, definitions, abbreviations, and synonyms for 17,000 words.

Because the first database has important data for decision-making, the detailed form and contents are shown in Table II. Control codes in Table II refer to the codes used to categorize the reviewed item as a strategic item. For example, the control code of a complete nuclear reactor can be assigned as 0A001, the code number of nuclear fuel can be assigned as 0A001.f, and so on. In addition, this database has system names in English and Korean, and has specifications related to complete nuclear reactors.

TABLE II: PRIMARY DATABASE FOR EXPERT SYSTEM

\begin{tabular}{cll}
\hline \hline No. & \multicolumn{1}{c}{ Structure } & \multicolumn{1}{c}{ Contents } \\
\hline 1 & Control Code & 0A001 \\
2 & Control Name & Complete Nuclear Reactors \\
3 & System Name & Light Water Reactor \\
4 & Related System & Reactor Coolant System \\
5 & $1^{\text {st }} / 2^{\text {nd }}$ System & $1^{\text {st }}$ System (Primary System) \\
6 & Name1 (Kor) & Korean Language Name1 \\
7 & Name2 (Kor) & Korean Language Name2 \\
8 & $\ldots$ & $\ldots$ \\
9 & Name1 (Eng) & Reactor \\
10 & Name2 (Eng) & Fission Reactor \\
11 & $\ldots$ & $\ldots$ \\
12 & Specification & Fission \\
& & Control... Reaction, \\
13 & Description (Kor) & Korean Language Description \\
14 & Description (Eng) & Various Types of Nuclear Reactor... \\
\hline \hline
\end{tabular}

Another important database for our systems is the terminology database. Its form is shown in Table III. 
TABLE III: TERMINOLOGY DATABASE FOR EXPERT SYSTEM

\begin{tabular}{c|c|c}
\hline \hline No. & Term (English version) & Term (Korean version) \\
\hline 1 & $1 /$ V detector & Translated to Korean_1 \\
$\ldots$ & $\ldots$ & $\ldots$ \\
44 & Absolute filter & Translated to Korean_44 \\
45 & Absolute manometer & Translated to Korean_45 \\
$\ldots$ & $\ldots$ & $\ldots$ \\
17784 & Zone Ring & Translated to Korean_17784 \\
\hline \hline
\end{tabular}

Note) Korean terms are not shown in this table

\section{Extracting the MRFs}

In this section, we extracted MRFs needed for each sub-step of the classification processes. Because MRFs are related to information level or type of document reviewed, these factors must be considered in making final decisions [6].

To extract MRFs, we selected 2,000 technical documents and related review reports in our web system (NEPS; the Nuclear Export Promotion System). Next, we categorized these documents according to characteristics or type of document. In the third step, we extracted MRFs by comparing document contents with contents of review reports. In the fourth step, we calculated the probability by MRFs in each document. We used a Bayesian inference equation for the probability calculation:

$$
\mathrm{p}(\mathrm{H} \mid \mathrm{E})=\frac{p(E \mid H) \times p(H)}{p(E \mid H) \times p(H)+p(E \mid \neg H) \times p(\neg H)}
$$

In this equation, event $\mathrm{E}$ means that the reviewed item has a type of MRFs, and event $\mathrm{H}$ means that the item is a strategic item. $\mathrm{p}(\mathrm{H})$ means the probability that the item is a strategic item. $\mathrm{p}(\mathrm{H} \mid \mathrm{E})$ means a probability that the item is a strategic item in the case of event $\mathrm{E}$.

As a result of the categorization, 2,000 technical documents could be categorized into 10 groups, such as manuals, analysis reports, specification documents, and requirements, as shown in Table IV.

TABLE IV: MRFS FOR 10 GROUPS

\begin{tabular}{|c|c|c|}
\hline Main Group & $\begin{array}{l}\text { Subgroup } \\
\text { (Document } \\
\text { Type) }\end{array}$ & MRFs \\
\hline \multirow{7}{*}{ Development } & $\begin{array}{l}\text { Analysis } \\
\text { Report }\end{array}$ & $\begin{array}{c}\text { TL Relation, Rough Procedure, } \\
\text { Detailed Procedure }\end{array}$ \\
\hline & Specification & $\begin{array}{c}\text { TL Relation, Input Value, } \\
\text { Equation, Calculation Results, } \\
\text { Material Name, Material Content }\end{array}$ \\
\hline & Requirement & $\begin{array}{l}\text { TL Relation, Analysis Methods, } \\
\text { Analysis Results }\end{array}$ \\
\hline & $\begin{array}{l}\text { Electronic } \\
\text { code }\end{array}$ & TL Relation \\
\hline & Data & $\begin{array}{l}\text { TL Relation, Data, Specification, } \\
\text { Graph, Drawing, Equation, Model }\end{array}$ \\
\hline & $\begin{array}{l}\text { Calculation } \\
\text { Note }\end{array}$ & $\begin{array}{l}\text { TL Relation, Input Value, Equation, } \\
\text { Calculation Results, } \\
\text { Material Name, Material Content }\end{array}$ \\
\hline & Drawing & $\begin{array}{c}\text { TL Relation, Drawing Type, } \\
\text { Numerical Value }\end{array}$ \\
\hline Use & Operation & $\begin{array}{l}\text { TL Relation, Operation Procedures, } \\
\text { Reactivity Control, Power Relation }\end{array}$ \\
\hline Use & Manual & $\begin{array}{c}\text { TL Relation, Rough Procedure, } \\
\text { Detailed Procedure, } \\
\text { Inspection Criteria }\end{array}$ \\
\hline $\begin{array}{c}\text { Not } \\
\text { Categorized }\end{array}$ & $\begin{array}{c}\text { General } \\
\text { Document }\end{array}$ & $\begin{array}{c}\text { TL Relation, Drawing, Numerical } \\
\text { Value, Input Value, Equation } \\
\text { Calculation Results }\end{array}$ \\
\hline
\end{tabular}

Note) TL means the strategic items
As shown in Table IV, the MRFs were categorized from one to nine, according to document type. As a representative group, electronic code had one factor, meaning that only this one factor may be used to determine whether the document in this group belongs to the strategic items. In contrast with the well-categorized groups, there were some documents that did not have these characteristics or exact forms, such as summary notes, letters, and descriptions. We could not categorize these into specific groups, and extracting MRFs from them was difficult. We gathered these documents into a general document group, and extracted various factors from the documents.

As the next step in MRFs extraction, we calculated the probability by MRF. In the first step, we re-checked the MRFs' existence in each document in the 10 groups, counted the number of MRFs included in each document, and re-confirmed whether each document concerned strategic items or not. Based on this checked information, we calculated the probability by MRFs for all groups using the Bayesian inference equation, as shown in Table V.

TABLE V: MRFS AND PROBABILITY FOR DRAWING GROUP

\begin{tabular}{|c|c|c|c|c|c|}
\hline Category & Type & \multicolumn{3}{|c|}{ Main Review Factors } & Prob. \\
\hline $\mathrm{D}$ & & $\mathrm{TL}$ & Drawing & Numeric & \\
\hline $\mathrm{E}$ & & Relation & Type & Value & \\
\hline V & $\mathrm{D}$ & $\mathrm{Y}$ & $\mathrm{Y}$ & $\mathrm{Y}$ & $100 \%$ \\
\hline $\mathrm{E}$ & $\mathrm{R}$ & $\mathrm{Y}$ & $\mathrm{Y}$ & $\mathrm{X}$ & $97 \%$ \\
\hline $\mathrm{L}$ & A & $\mathrm{Y}$ & $\mathrm{X}$ & $\mathrm{Y}$ & $100 \%$ \\
\hline $\mathrm{O}$ & W & Y & $\mathrm{X}$ & $\mathrm{X}$ & $88 \%$ \\
\hline $\mathrm{P}$ & I & $\mathrm{X}$ & $\mathrm{Y}$ & $\mathrm{Y}$ & $38 \%$ \\
\hline M & $\mathrm{N}$ & $X$ & $\mathrm{Y}$ & $\mathrm{X}$ & $1 \%$ \\
\hline $\begin{array}{l}E \\
N\end{array}$ & $\mathrm{G}$ & $X$ & $X$ & $\mathrm{Y}$ & $12 \%$ \\
\hline $\mathrm{T}$ & & $\mathrm{X}$ & $\mathrm{X}$ & $\mathrm{X}$ & $0 \%$ \\
\hline
\end{tabular}

Table $\mathrm{V}$ is an example of the Drawing group from Table IV. ' $Y$ ' means that the reviewed document included MRFs, and ' $\mathrm{X}$ ' means that the reviewed document did not. For example, if the drawing is related to the strategic items list (third column), the drawing form is CAD (fourth column) and includes numerical values, the strategic items' probability for a drawing document can be $100 \%$. Although other examples are not described in Table V, the same methods can be used to calculate the probability of strategic items.

\section{Extracting EFs}

The MRFs in Section $C$ focus on the technical aspects of the reviewed documents. However, there are other important criteria or factors that must be considered in classifying the strategic items. These are the EFs. There are no definite criteria or forms for these factors, and they may be abstruse, ambiguous, and qualitative. Thus, all of the NSG member states have difficulties applying these factors in classifications.

We sought to change these ambiguous criteria into more clear, quantitative, and applicable criteria. To do this, we first collected the ambiguous criteria through interviewing the experts at our institute, and extracted some clues and evidence by re-analyzing 2,000 technical documents and related review reports. Next, we compiled meaningful things from the collected ambiguous criteria, extracted clues, and evidence, and designated the meaningful items as EFs. In the third step, we calculated the probability by EFs. The first step 
for this was an expert survey; the seven questions in Table VI were given to the experts. Because each question is for a significant feature, such as political aspects, technical aspects, and importer's aspects, each expert scored the degree of contribution, from zero to one, for each EFs based on each expert's experience or know-how. After the survey, we averaged the surveyed scores as shown in Table VI. In this Table IV, the value in the Yes column is the degree of belief by which the reviewed item can be categorized as a strategic item. The value in the No column is the degree of belief by which the reviewed item can be categorized as a non-strategic item.

TABLE VI: EDP FACTORS FOR CLASSIFICATION

\begin{tabular}{l|c|c}
\multicolumn{1}{c|}{ EDP Factors (EFs) } & Yes & No \\
\hline Is Customized Production necessary? & 0.62 & 0.33 \\
\hline Is there the direct relationship for nuclear material? & 0.60 & 0.32 \\
\hline Is Reviewed Item component parts? & 0.42 & 0.63 \\
\hline$\cdots$ & $\cdots$ & $\cdots$ \\
\hline \hline
\end{tabular}

The first question for the EFs was evaluating whether the reviewed item is the customized product. The reason that this EFs must be evaluated is that the components, equipment, or techniques related to nuclear activities are generally not ready-made products. The second question for the EFs evaluates the relationship with nuclear material. Strategic items generally have very close relationships with nuclear materials, because nuclear materials such as uranium and plutonium can be directly used to produce nuclear weapons. So, this factor must be considered in classification. The third question was for evaluating whether the reviewed item was a complete or integrated component. In most international regimes, including NSG, there have been many arguments about the control of component parts or equipment parts. However, the regimes strongly request that all of the member states control or manage parts of components or equipment, if those parts of components or equipment could be used for WMD. Thus, we designated this factor as an important EFs. The other EFs not described in the Table IV relate to the volume of technical documents, conceptual information for strategic items, physical items, and technologies not listed in the Minster's Regulations.

\section{E. Process Optimization and Expert System Development}

To optimize and develop the expert systems, we integrated generalized processes, databases, MRFs, and EFs as described in the previous sections. Then, we built two optimized processes for physical items and technology, as shown in Fig. 3 and Fig. 4, respectively.

As shown in Fig. 3, the physical item process has many checking or matching stages. However, there are three key stages: name matching, function matching, and specification matching. There is another important stage for EFs checking, although it is an optional stage and can be omitted at the reviewer's discretion.

This process is generally more definite and clearer than the technology process shown in Fig. 4, because matching for an item's name, function, and specification are more definite than the stages of the technology process, such as open source checking, basic science checking, and evaluating the level and depth of the technology.

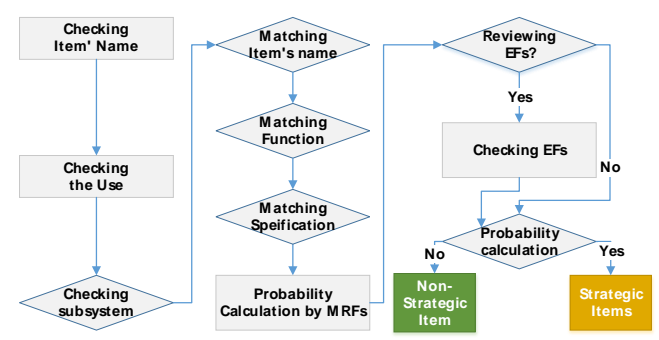

Fig. 3. Review process for the physical items.

Fig. 4 shows the classification process for technology. This process has many checking and matching stages, similar to the physical item process. For example, this process checks for item names related to the technology, use, subsystems, and EFs. However, there are also special checking stages in technology classification, such as open-source checking. The reason for this checking is that NSG and other international regimes generally do not designate open-source or public-domain information as strategic items. Thus, this stage is important in the classification process for technologies.

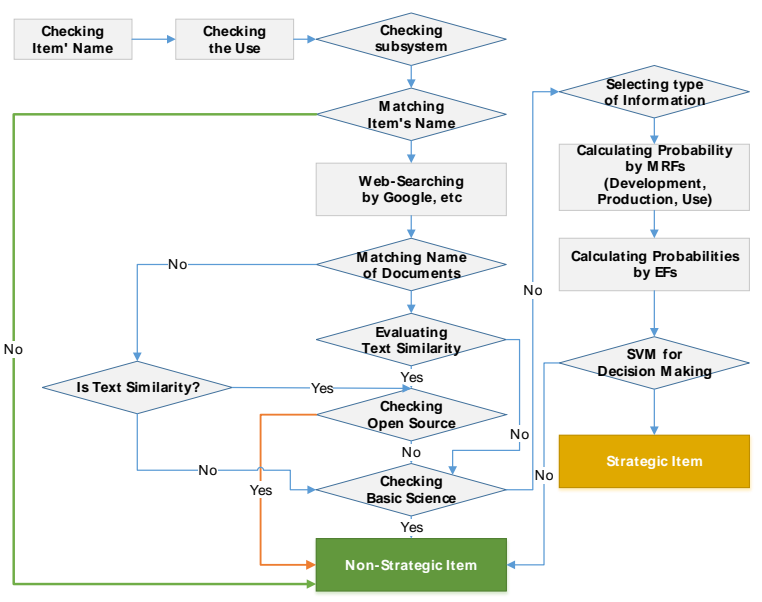

Fig. 4. Review process for technology.

The methods for open-source checking were text-similarity evaluation methods, such as cosine similarity in the equation below [8], where Di means the frequency of specific text in the newly reviewed document, and $Q j$ means the frequency of specific text in the searched documents by web searching.

$$
\cos \left(D_{i}, Q_{j}\right)=\frac{\sum_{k=1}^{t} d_{i k} q_{j k}}{\sqrt[2]{\left(\sum_{k=1}^{t} d_{i k}^{2} \times \sum_{k=1}^{t} q_{i k}^{2}\right)}}
$$

The comparison process in the expert system is as follows, which was developed in this research. First, the system user copies the titles of the newly reviewed documents, and searches for articles that have the same or similar names using the Google search engine. Second, the user saves the texts of the reviewed documents into the buffer memory of the system, and saves text of the searched documents into another buffer memory. Third, the user operates the comparison module to evaluate the similarity between reviewed documents and searched documents. Then, the system gives the results of a similarity comparison between two documents to the user.

In addition, there is another special checking stage in 
contrast with that of the physical item process. This is information type selection and a probability calculation. There is also a probability calculation stage in the physical item process, but the detailed method is different between the processes. In the physical item process, the reviewer only has to check or match the name, function, and specification in the reviewed items, based on the list of the Minister's Regulation. Then, the probability can be calculated automatically by the same level of contribution for strategic items, such as $33 \%$. However, in the technology process, the reviewer must check the information type and evaluate the level of depth of the contents. That is, the probability calculation process for technology is more complex than that for physical items.

In this aspect, we improved the probability calculation process by integrating MRFs checking in section $\mathrm{C}$ and EFs checking in section $\mathrm{D}$, except for physical items.

Next, we randomly sampled 149 technical documents and performed reviews based on the MRFs and the EFs in our 2,000 documents. Reviews of physical items were omitted because the number of physical items was less than the number of technical documents and their review factors are more definite and clearer than those for technology, as mentioned above. As an example, the relationship with the development of nuclear strategic items is an ambiguous MRFs, because some experts think that almost all of the nuclear items and documents are related to each other, whereas others think that the only specific nuclear items mentioned in the document are related.

All of the sample data in this simulation had been classified already. That is, all of the sample data had answers for strategic items or not. The experts who participated in this simulation did not know those answers, and reviewed all of the documents based on each expert's knowledge, opinion, and know-how. Three experts participated in this simulation and only reviewed documents according to the EFs.

The sample data were slightly biased in this simulation as below. In all, 24 documents consisting of 12 strategic items and 12 non-strategic items were used as a training data set for the support vector machine (SVM) [9, 10], and the other 125 documents consisting of 36 strategic items and 89 non-strategic items were used as the evaluation data set.

We reviewed technical documents by checking MRFs. If the threshold or probabilities by MRFs was 0.5, using a MRFs probability in Table $\mathrm{V}$, we can designate the reviewed document as a strategic item. In our review simulation, we could confirm that 110 of the 125 documents corresponded with the earlier answer sets.

We also performed the same review by applying EFs. Each item has a different score according to the EFs in Table VI. When the sum of scores is greater than the threshold, the system classifies the reviewed item as a strategic item. The optimum threshold was 10.9. In this case, we could confirm that 102 of 125 documents corresponded with the earlier answer sets.

That is, the EFs could not be ignored, although a classification according to MRFs was more precise or obvious than the EFs, because both of them have been key factors in implementing classifications. Moreover, there were also many cases where MRFs could not distinguish, but the EFs could. Thus, an appropriate approach could require optimizing the two classification results to improve the accuracy of classification.

Next, we tried to integrate the two factors into a single evaluation factor. An ensemble classification is popular, but it usually requires more than two classifiers that differ from each other. Because we had just two classifiers, we applied a SVM, which is a binary classifier, based on optimization theory from decision making. In this study, SVM ${ }^{\text {light }}$ [9] was used to train an integrated classifier using 24 documents. Scores according to the EFs were normalized and they ranged from zero to one before applying SVM.

As a result of the integration, the training error was $4.17 \%$ and the classifier classified 111 documents precisely among the 125 documents. This shows that the integrated classifier was slightly better than considering just one of the two types of review factors. Thus, we applied this classification model to the expert system in Table VII.

TABLE VII: RESULT BY APPLYING SVM FOR DECISION MAKING

\begin{tabular}{l|c|c}
\hline Method & $\begin{array}{c}\text { Corresponding } \\
\text { rate }\end{array}$ & $\begin{array}{c}\text { Training } \\
\text { Error }\end{array}$ \\
\hline Case by applying Main Review Factors & 0.880 & - \\
\hline Case by applying EDP Factors & 0.816 & - \\
\hline Case by applying SVM & 0.888 & $4.17 \%$ \\
\hline \hline
\end{tabular}

In final stage, we developed the expert systems for physical items and technologies, based on the previous results [11]. We used MS-SQL for DBMS and JAVA as the development language. Each system adopted the property-sheet concept for its user interface. Each sheet provides necessary information on each sub-stage for decision making by users.

The expert system for physical items is shown in Fig. 5, and this system includes all of the processes from Fig. 3 and the relevant database for the classification. This system can automatically present information or check points to the users, and the user can confirm all information given by the system. In the final stage of this system, the system gives a summary of the checked or matched information to the user, and the user can re-confirm or modify the summary for the final decision-making.

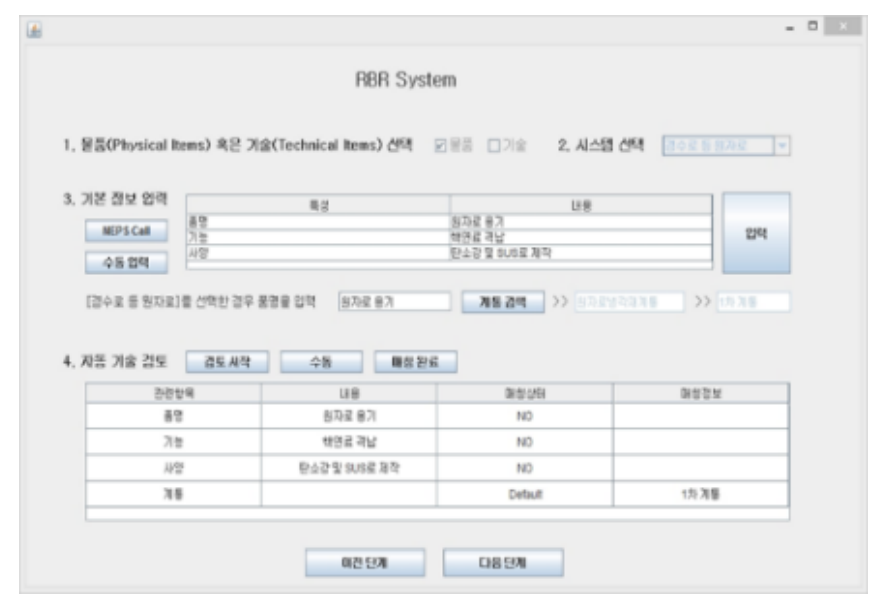

Fig. 5. Expert system for physical items.

The expert system for technology is shown in Fig. 6. The operation process of this system is similar to that of the physical items. However, this system has some additional sub-stages, as mentioned previously, such as open source checking, evaluation for level of depth of the contents, and a 
probability calculation by SVM.



Fig. 6. Expert system for technology.

That is, the technology expert system needs the user's experience or know-how for the classification or searching, compared to the physical item's expert system. After the user checks or confirms all questions given by this system, the system gives a summary of checked or confirmed information, and the user can re-confirm or modify the summary for final decision-making.

\section{CONCLUSIONS}

As mentioned previously, there is some probability that different experts will make different decisions for the same or similar reviewed items, because the criteria of the Minister's Regulation and the NSG guideline are ambiguous and qualitative. This obviously can be harmful to consistency or the objectivity of classification results. Although we always use consultation meetings to solve those problems for ambiguous items, this takes a long time and much effort and we still cannot completely solve all of these problems.

Thus, there is a need to minimize the probability of making errors in classifications, although not all of the problems can be resolved completely. So, we generalized the classification processes for physical items and technologies, developed a database, extracted MRFs and EFs, and optimized the classification processes. Then, we developed two expert systems based on these researched results.

As these systems are the first systems in the export control field, we will consistently use the systems for classifications. We will also assess the consistency and objectivity of results, and the efficiency of the classification process through those efforts.

However, there are some issues that must be improved in the expert systems developed, such as the lack of categorized groups for documents and the applicability of MRFs and EFs to the reviewed documents. That is, the systems have limitations in the classification, because the 10 groups in section $\mathrm{C}$ cannot cover all documents for all nuclear systems.
Applicability issue of MRFs and EFs may be caused by a lack of factors in some document groups or by doubts about the representative nature of extracted factors for the reviewed documents. The expert systems may not always give reliable review results to the user because of these issues. Thus, we will build more groups through analyzing all of the documents in our web system, and we will extract more accurate or representative factors that can be applied to all document reviews, based on the groups determined.

\section{REFERENCES}

[1] Guidelines for Nuclear Transfers, INFCIRC/254/Rev.12/Part1, IAEA 2013

[2] Foreign Trade Act, Ministry of Trade, Industry \& Energy of ROK, 2013

[3] Minister's Regulation for the Export and Import of Strategic Goods, Ministry of Trade, Industry \& Energy of ROK, 2014

[4] M. Firebaugh, Artificial Intelligence: A Knowledge-Based Approach, Boyd \& Fraser Boston

[5] J. W. Tae and D. H. Shin, "A Study on the systematization of commodity classification," Transaction of Korean Nuclear Society Autumn Meeting, Oct. 25-26, 2012.

[6] S. M. Han, S.-H.Yang, J.-W. Tae, and D. H. Shin, "Research on the re-establishment of the classification criteria of strategic items," Transaction of Korean Nuclear Society Spring Meeting, May 29-30, 2014.

[7] NSG Trigger List Handbook, U.S. Department of Energy/National Nuclear Security Administration.

[8] A. N. Aizawa, "An information-theoretic perspective of tf-idf measures," Inf. Process. Management, vol. 39, no. 1, pp. 45 - 65, 2003

[9] T. Joachims, "Making large-Scale SVM Learning Practical," Advances in Kernel Methods - Support Vector Learning, B. Schölkopf, C. Burges, and A. Smola, eds., MIT-Press, 1999.

[10] T. Joachims, "Text categorization with support vector machines: learning with many relevant features," in Proc. the European Conference on Machine Learning (ECML), pp. 137-142, 1998.

[11] M. Dekker, "Nikolopoulos and C. Expert systems: Introduction to first and second gener-ation and hybrid knowledge based systems," Inc., 1997.

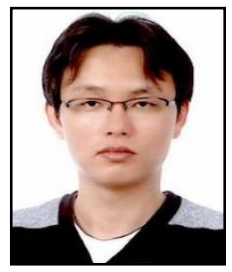

Dong Hoon Shin was born in the Republic of Korea in 1976. He received his master's degree in medical physics from Catholic University and $\mathrm{PhD}$. degree in nuclear engineering from Seoul National University, Republic of Korea, in 2007. He is working as a senior researcher in Korea Institute of nuclear nonproliferation and control. His research interests include the data and text mining, artificial intelligence,

image similarity, and applications for nuclear nonproliferation policy and implementation.

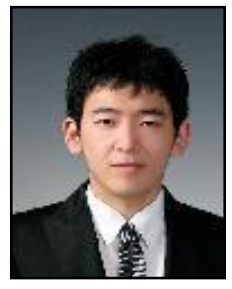

Jae-Woong Tae was born in Pusan, Republic of Korea in September 1982. He received the MS degree from the Department of Mathematical Sciences, Korea Advanced Institute of science and technology (KAIST), Daejeon, Republic of Korea, in 2010. He is a researcher in Korea Institute of Nuclear Nonproliferation and Control (KINAC). His current research interests include data and text mining, computer vision. 